\title{
Electric and Magnetic Field Effects on Electronic Structure of Straight and Toroidal Carbon Nanotubes
}

\author{
C. G. Rocha, M. Pacheco ${ }^{\dagger}$, Z. Barticevic ${ }^{\dagger}$, and A. Latgé* \\ *Instituto de Física, Universidade Federal Fluminense \\ Campus da Praia Vermelha, Av. Litorânea, s/n, Boa Viagem, 24210-340 Niterói, Rio de Janeiro, Brazil \\ ${ }^{\dagger}$ Departamento de Física, Universidad Técnica F. Santa Maria \\ Casilla, 110-V, Valparaiso, Chile
}

Received on 31 March, 2003

\begin{abstract}
Nanotubes have been proved as promising candidates for many technological applications in the nanoscale word and different physical properties have been studied and measured along the few recent years. Here we investigate the role played by external magnetic and electric fields on the electronic properties of toroidal and cylindrical straight carbon nanotubes. A single- $\pi$ band tight-binding Hamiltonian is used and two types of model-calculations are adopted: real-space renormalization techniques, based on Green function formalism, and straight diagonalization calculation. Both electric and magnetic fields may be properly applied, in different configurations, to modify the energy spectra and transport properties, providing metal-insulator transitions for particular tube geometries.
\end{abstract}

\section{Introduction}

Perfect carbon rings, stabilized only by van der Waals forces[1, 2], were reported recently and suggest an experimental evidence of circular molecules named Toroidal Carbon Nanotubes (TCNs). They may be used as a model material for low dimensional molecular conductors and their properties are mainly governed by geometrical characteristics. In transport measurements, carbon rings and/or tubes are usually placed in metal electrodes, oxide substrate, gate potentials and magnetic fields[3, 4]. These environments give rise to extra potentials on the carbon atoms, affecting and changing both electronic and transport properties. Due to its very peculiar electronic property, one may induce metal-insulating transitions in carbon nanotube-based devices, for instance, by exposing the tubes to external fields. In that sense, carbon nanotubes may be used as a nanometric electromechanical switch.

The carbon-ring structure may be described as long rolled graphite sheets bent around in the form of tori, satisfying simultaneously transversal and longitudinal periodical boundary conditions. The presence of a homogeneous magnetic field threading the annular system is described within the Peierls-phase approximation[5, 6]. In a previous work we have investigated how a magnetic field modify the electronic properties of torus nanotubes[7]. A single $\pi$-band tight-binding Hamiltonian was used and real-space renormalization techniques, within the Green function formalism, was adopted. The local density of states (LDOS) was shown to exhibit a sequence of well defined peaks, shifted in the presence of the axial magnetic field. It may also be shown that the "central gap size of a semiconductor TCN is periodically modulated by increasing the magnetic flux $(\phi)$ thread- ing the torus cross section due to Aharonov-Bohm oscillations on the LDOS.

Interesting changes in the electronic properties of carbon nanotubes may also be observed when an electric field is applied along different directions. For instance, an uniform transverse electric field may be viewed as being generated by two capacitor planes displayed on and up a carbon tube[8,9]. The presence of the electric field modifies the on-site energies of carbon atoms following a linear interpolation for the potential energy difference along the nanotube diameter. The transversal field may be used then to modulate the gap, effective mass and to increase the density of states at the Fermi level[10].

The electronic properties of a carbon torus under applied fields in the Hall-field configuration (magnetic and electric field applied perpendicular and parallel to the torus plane, respectively)[11] are also discussed. The renormalization of the Green functions becomes quite difficult in this case since all the undressed locators are modified due to the presence of the electric field. Therefore, to obtain the energy eigenvalues, we diagonalize numerically the tight binding Hamiltonian matrix. The energy spectra as a function of electric field strength is presented in order to observe the evolution of the states when the field intensity is increased. We investigated the conditions under which it is possible to induce metal-insulating transitions by applying both external fields.

\section{Results and discussion}

The Hamiltonian of the $\mathrm{CN}$ is entirely treated in the real space and one considers a sequence of connected rings forming the tubes, described within a single $\pi$-band tight-binding 
approximation. This treatment restrict our results to the energy range near the Fermi level. The carbon torus is constructed closing the tube at the axial direction considering periodic boundary conditions. For the sake of simplicity we restrict our analysis on zigzag/armchair $(n, 0, N, N)$ and armchair/zigzag $(n, n, N, 0)$ torus, with $N$ being the number of connected rings closed in a torus shape[8].

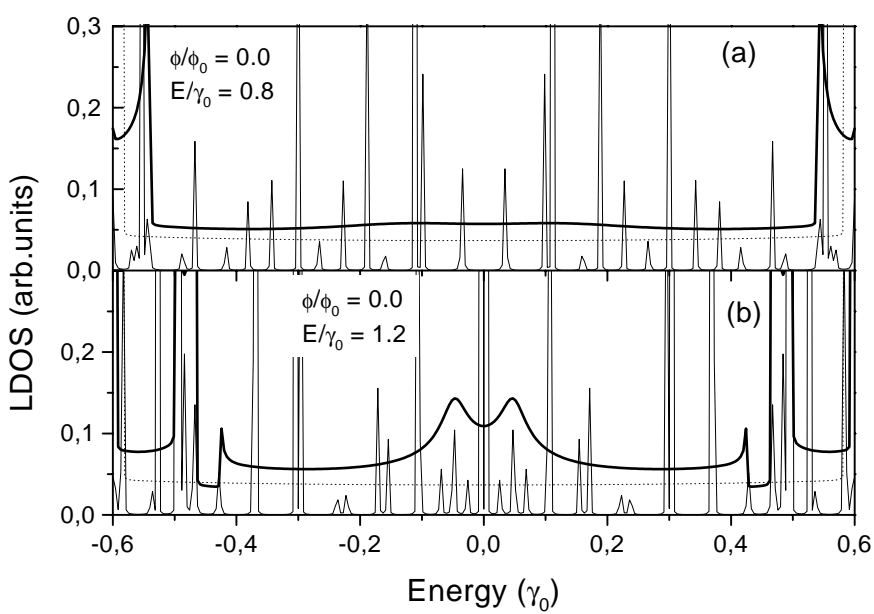

Figure 1. LDOS as a function of the energy (written in terms of the hopping $\gamma_{0}$ ) for two electric field intensities: $E / \gamma_{0}=$ (a) 0.8 and (b) 1.2. Bold and dotted lines are the results for a $(5,5) \mathrm{CN}$ with and without electric field, respectively. The set of peaks corresponds to the LDOS of a $(5,5,50,0) \mathrm{TCN}$ in the presence of the corresponding electric fields.

Results for the LDOS of a straight $(5,5)$ armchair CN and a $(5,5,50,0)$ armchair/zigzag TCN are shown in Fig. 1 for two transversal electric field intensities. The typical pattern of the density of states of a $\mathrm{CN}$ in the absence of electric fields (shown in dotted line) is clearly distorted and it suffers an enhancement near the Fermi level $\left(E_{F}=0\right)$. These features are associated with the flattening and mixing of the subbands as the field is increased[10]. A similar behavior is also found for the toroidal structure, remarking that the LDOS is given now by a sequence of well defined peaks, due to the TCN finite size nature, shifted in the presence of the field.

An interesting point to note is the fact that armchair CNs are always metallic, even at very large electric fields, within this tight-binding picture. Contrary to the present result, Xin et al.[9] have shown that semiconducting induced behavior may be attained if the electric-field is also included in the off-diagonal terms of the Hamiltonian.

On the other hand, we found that applying an axial magnetic field, such a transition may be induced, as shown in Fig. 2 for a $(5,5)$ armchair $\mathrm{CN}$, in an electronic transitionlike diagram. The critical electric-field energy is defined here as the electric field value for which an insulating transition occurs, for a given magnetic flux (written in terms of $\left.\phi_{0}=h / e\right)$. One may notice the oscillatory behavior due to the applied magnetic field, characteristic of the annular geometry. Otherwise, for zigzag $\mathrm{CNs}$, the opening of the energy gap may be induced applying merely an electric field as depicted in Fig. 3.

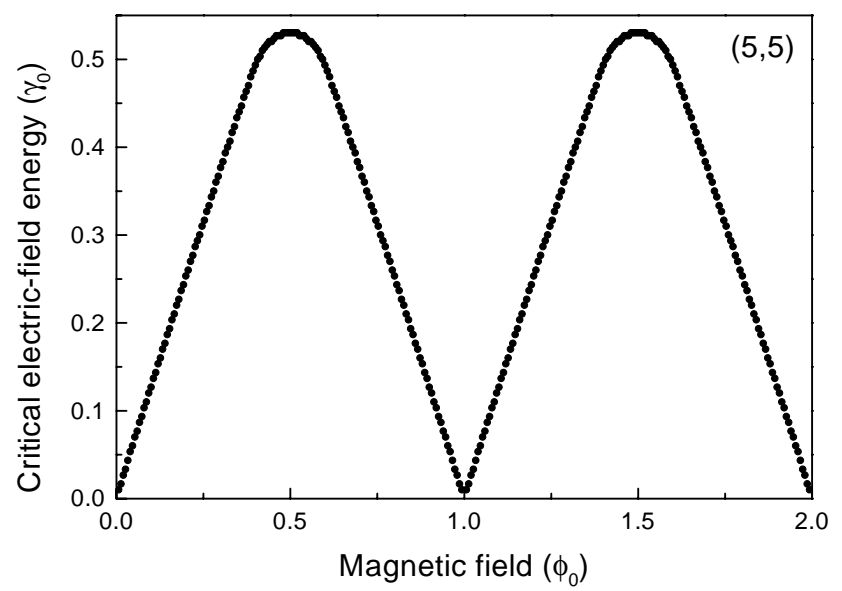

Figure 2. Metal-insulator transition diagram of the magnetic flux and the critical effective electric-field energy at $E_{F}=0$, for a $(5,5) \mathrm{CN}$.

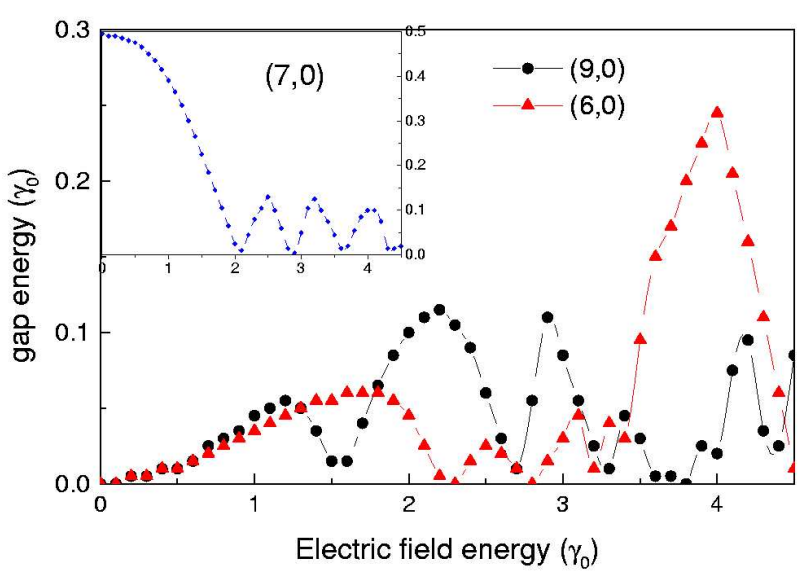

Figure 3. Dependence of the gap energy on the electric field strength for $(9,0)$ (circles), $(6,0)$ (triangles), and $(7,0)$ (inset) CNs.

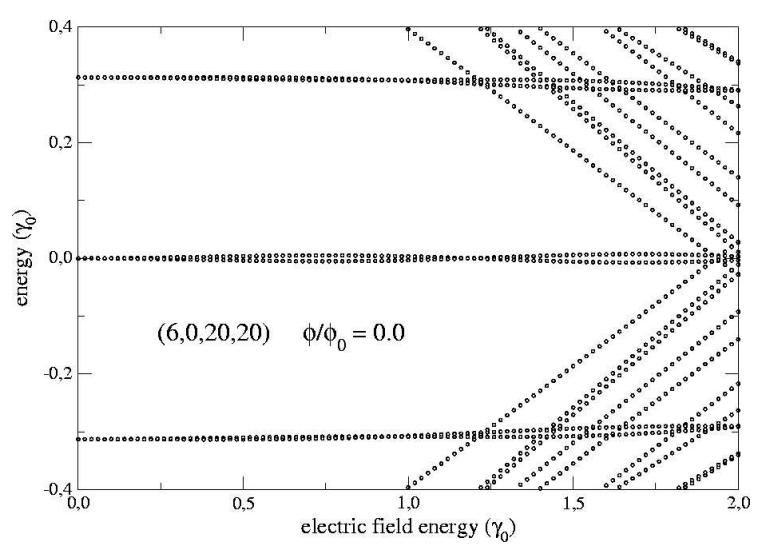

Figure 4. Energy spectra as a function of the electric field energy for a $(6,0,20,20) \mathrm{TCN}$ and null magnetic field.

Concerning now with TCN structures under external fields in the Hall configuration, we present the energy spectra of $(6,0,20,20)$ and $(8,0,20,20)$ structures, in Figs. 4 and 
5 , respectively, as a function of the effective electric field energy. For the "metallic" example shown in Fig. $4(\phi=0)$, one notices that the eigenstates close to the Fermi level do not suffer the effects of the electric field, except for the quite subtle lifting of the degenerate states.

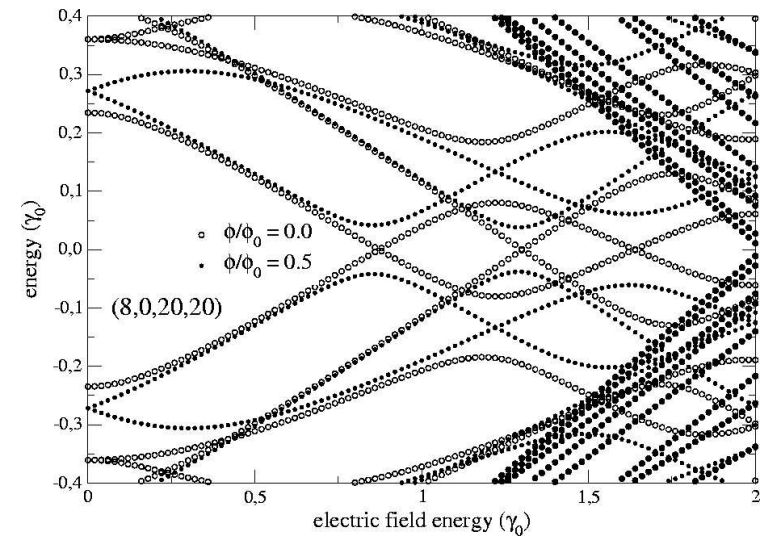

Figure 5. Energy spectra versus electric field energy for a $(8,0,20,20) \mathrm{TCN}$ and magnetic flux $\phi / \phi_{0}=0$ and 0.6 (open and solid circles, respectively).

The other chosen example exhibits clearly the energy spectrum dependence on both fields. The main effect of the magnetic flux $\left(\phi / \phi_{0}=0.6\right)$ is to lift the double degeneracy of the eigenstates and also to allow the formation of anticrossing curves near the Fermi energy. The consequence is that no metal-insulator transition is allowed within the investigated electric-field energy range. A detailed study of the role played by symmetries of the zigzag/armchair annular structures is now in process as well as a comparison between the gap evolution of the TCNs under different field configurations. This would certainly improve our considerations about the external field responses.

\section{Conclusion}

Carbon nanotubes and correlated structures (in particular the studied toroidal nanotubes) do present themselves a variety of electronic properties depending upon their intrinsic geometric formation. Adding external fields, one may intentionally modify these properties and modulate their physical responses. We believe that a better understanding of the physics of carbon nanostructures, even within a simple picture as the one adopted here, should help us to propose their utilization in real nanodevices.

\section{Acknowledgments}

This work was partially supported by CNPq, FAPERJ, CYTED, Fondecyt 1010429, and P99-135F.

\section{References}

[1] J. Liu, et. al., Nature 385, 780 (1997).

[2] R. Martel, H. R. Shea, and P. Avouris, Science 398, 299 (1999).

[3] C. Dekker, Phys. Today 52 (5), 22 (1999).

[4] J.-O. Lee, et. al., Solid State Comm. 115, 467 (2000).

[5] S. Roche, G. Dresselhaus, M. S. Dresselhaus, and R. Saito, Phys. Rev. B 62, 16092 (2000).

[6] H. Ajiki, and T. Ando, J. Phys. Soc. Jpn. 65, 505 (1996).

[7] A. Latgé, C. G. Rocha, L. A. L. Wanderley, M. Pacheco, Z. Barticevic, and P. Orellana, Phys. Rev. B67 155413 (2003).

[8] Y. Kim, and K. Chang, Phys. Rev. B 64, 153404 (2001).

[9] X. Zhou, et. al., J. Phys.: Condens. Matter 13, L635 (2001).

[10] Y. Li, S.V. Rotkin, and U. Ravaioli, Nano Letters 3 (2), 183 (2003).

[11] Z. Barticevic, G. Fuster, and M. Pacheco, Phys. Rev. B 65, 193307 (2002). 